\title{
Modeling and Simulation of Reaction Environment in Photoredox Catalysis: A Critical Review
}

\author{
Gabriela Xavier de Oliveira, Jéssica Oliveira de Brito Lira, Humberto Gracher Riella, \\ Cíntia Soares * and Natan Padoin*
}

Laboratory of Materials and Scientific Computing (LabMAC), Department of Chemical and Food Engineering (EQA), Federal University of Santa Catarina (UFSC), Florianópolis, Brazil

\section{OPEN ACCESS}

Edited by:

Evgeny Rebrov,

University of Warwick,

United Kingdom

Reviewed by:

Ekaterina Kozlova,

Boreskov Institute of Catalysis (RAS),

Russia

Rufat Abiev,

Saint Petersburg State Institute of

Technology, Russia

*Correspondence:

Cíntia Soares

cintia.soares@ufsc.br

Natan Padoin

natan.padoin@ufsc.br

Specialty section:

This article was submitted to Microfluidic Engineering and Process

Intensification,

a section of the journal

Frontiers in Chemical Engineering

Received: 03 October 2021

Accepted: 23 November 2021

Published: 14 January 2022

Citation:

de Oliveira GX, Lira JOdB, Riella HG

Soares $C$ and Padoin N (2022)

Modeling and Simulation of Reaction Environment in Photoredox Catalysis:

A Critical Review.

Front. Chem. Eng. 3:788653.

doi: 10.3389/fceng.2021.788653
From the pharmaceutical industry's point of view, photoredox catalysis has emerged as a powerful tool in the field of the synthesis of added-value compounds. With this method, it is possible to excite the catalyst by the action of light, allowing electron transfer processes to occur and, consequently, oxidation and reduction reactions. Thus, in association with photoredox catalysis, microreactor technology and continuous flow chemistry also play an important role in the development of organic synthesis processes, as this technology offers high yields, high selectivity and reduced side reactions. However, there is a lack of a more detailed understanding of the photoredox catalysis process, and computational tools based on computational fluid dynamics (CFD) can be used to deal with this and boost to reach higher levels of accuracy to continue innovating in this area. In this review, a comprehensive overview of the fundamentals of photoredox catalysis is provided, including the application of this technology for the synthesis of addedvalue chemicals in microreactors. Moreover, the advantages of the continuous flow system in comparison with batch systems are pointed out. It was also demonstrated how modeling and simulation using computational fluid dynamics (CFD) can be critical for the design and optimization of microreactors applied to photoredox catalysis, so as to better understand the reagent interactions and the influence of light in the reaction medium. Finally, a discussion about the future prospects of photoredox reactions considering the complexity of the process is presented.

Keywords: photoredox catalysis, flow photochemistry, micro-structured reactors, computational fluid dynamics (CFD), process intensification

\section{HIGHLIGHTS}

- Photoredox catalysis is a powerful tool in the field of organic synthesis.

- Overview of applications for the synthesis of added-value chemicals using photoredox catalysis.

- Photoredox catalysis in microreactors: CFD modeling as a pivotal approach towards a better understanding of the process.

- Future perspectives for the optimization of photoredox catalysis process design by photon transport simulation and CFD. 


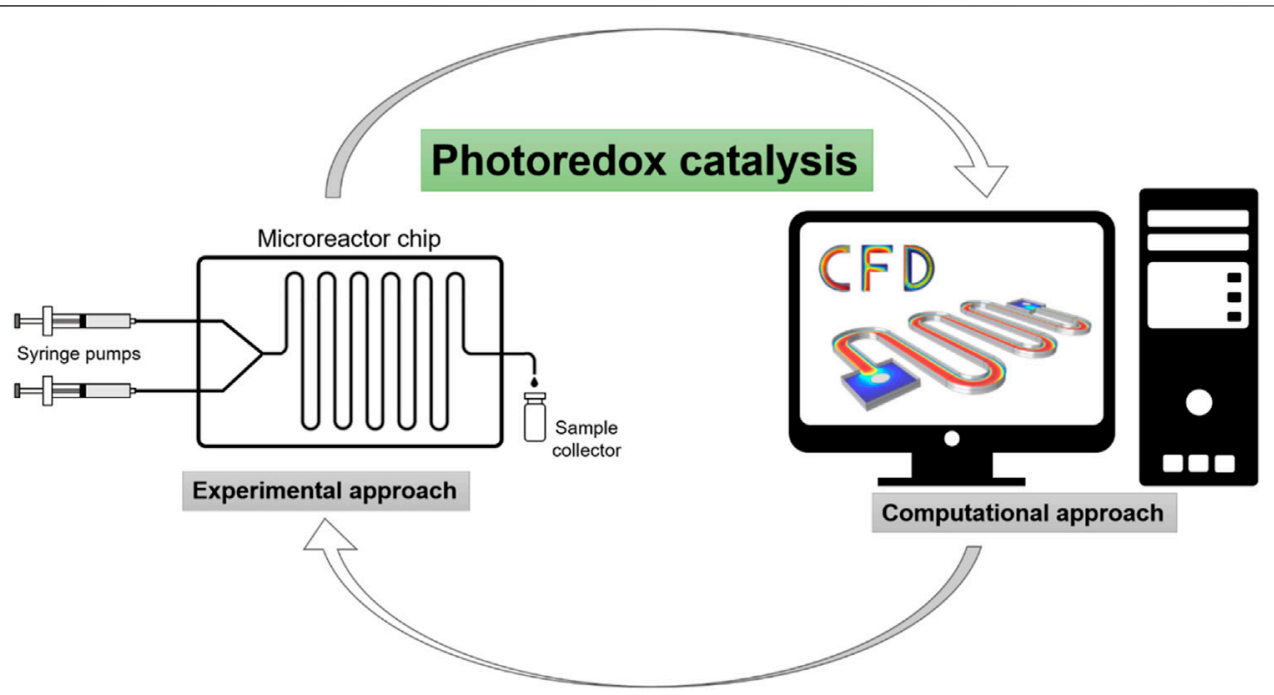

GRAPHICAL ABSTRACT

\section{INTRODUCTION}

The future development of synthetic organic chemistry involves environmentally friendly and sustainable alternatives in terms of energy to promote chemical transformations, using "light" as an abundant and renewable energy source (Narayanam and Stephenson, 2011; Shaw et al., 2016). Photochemical reactions are based on the use of light (ultraviolet, visible or solar) to provide the activation energy to induce the synthesis of a target molecule from a simple substrate. In this way, researchers explore ways to efficiently collect and use light energy for the activation of organic molecules (Noël, 2017; Crisenza and Melchiorre, 2020).

In the last decade, photoredox catalysis has emerged as a versatile platform for catalytic methodologies for organic synthesis, using photons of visible light as a reagent without tracking. In a general sense, this approach is based on the ability of metallic complexes and organic dyes to get involved in single electron transfer (SET) processes with the organic substrate after photoexcitation with visible light (Nicholls et al., 2016). This technology has become an indispensable tool in the search for new biologically active compounds for applications in medicine, fine chemical and pharmaceutical industries, as well as in many other fields of science, materials and the environment (Aillet et al., 2013; Loubière et al., 2016). Moreover, recent progress in the use of photoredox catalysis as a new conceptual approach to mediate synthetic organic reactions using visible light has been promoted by the combined efforts of three research groups: MacMillan (Shaw et al., 2016), Stephenson (Narayanam and Stephenson, 2011), Yoon (Schultz and Yoon, 2014) and many other researchers.

However, this growing interest in photochemistry has also brought to light some old and unresolved challenges such as reproducibility, scale and efficiency (Corrigan et al., 2016). Most of these issues are associated with the complexity of photochemical processes. The scale increase is hampered by the photon transport attenuation effect (Bouguer-Lambert-
Beer law). Excessive radiation can be a relevant issue in using larger reactors, as reaction times would increase substantially and would also result in the formation of undesired by-products (Cambié et al., 2016). However, these problems associated with batch photochemistry can be overcome with the use of continuous flow systems.

Continuous flow microreactors have recently emerged as promising instruments for chemical synthesis. Due to the small size of the reaction channel, the microreactors favor the heat and mass transport, allow precise control of the reaction and also allow extensive penetration of light (even in concentrated solutions) becoming quite advantageous in micro (photo) chemical reactions. Several studies report that this technology is also suitable and has many benefits for photochemical transformations, increasing interest in flow photochemistry (Coyle and Oelgemöller, 2008; Knowles et al., 2012; Jensen et al., 2014; Su et al., 2014; Loubière et al., 2016; Straathof et al., 2016; Su et al., 2016).

Despite many studies aimed at the application of microreactors for organic synthesis, this approach is mostly laboratory research (Weissman and Anderson, 2015). The experimental methods provide an overview of the process and do not allow a local analysis inside the reactor. To achieve higher levels of accuracy and more rigorous and detailed analysis, microscopic models based on transport phenomena must be employed. In this sense, computational fluid dynamics (CFD) can be highlighted as a useful simulation tool.

These methods simulate the reactive flow within the entire reactor, considering the coupling of all phenomena that occur (fluid flow, mass and heat transport, and photon transport) (Oliveira de Brito Lira et al., 2021). Modeling organic reactions can be challenging due to complex reaction profiles (Armstrong et al., 2019). Therefore, once the model is validated, relevant information regarding the reactive flow in the microchannels and results regarding the yield and selectivity of the photoredox-type reaction can be easily obtained. 


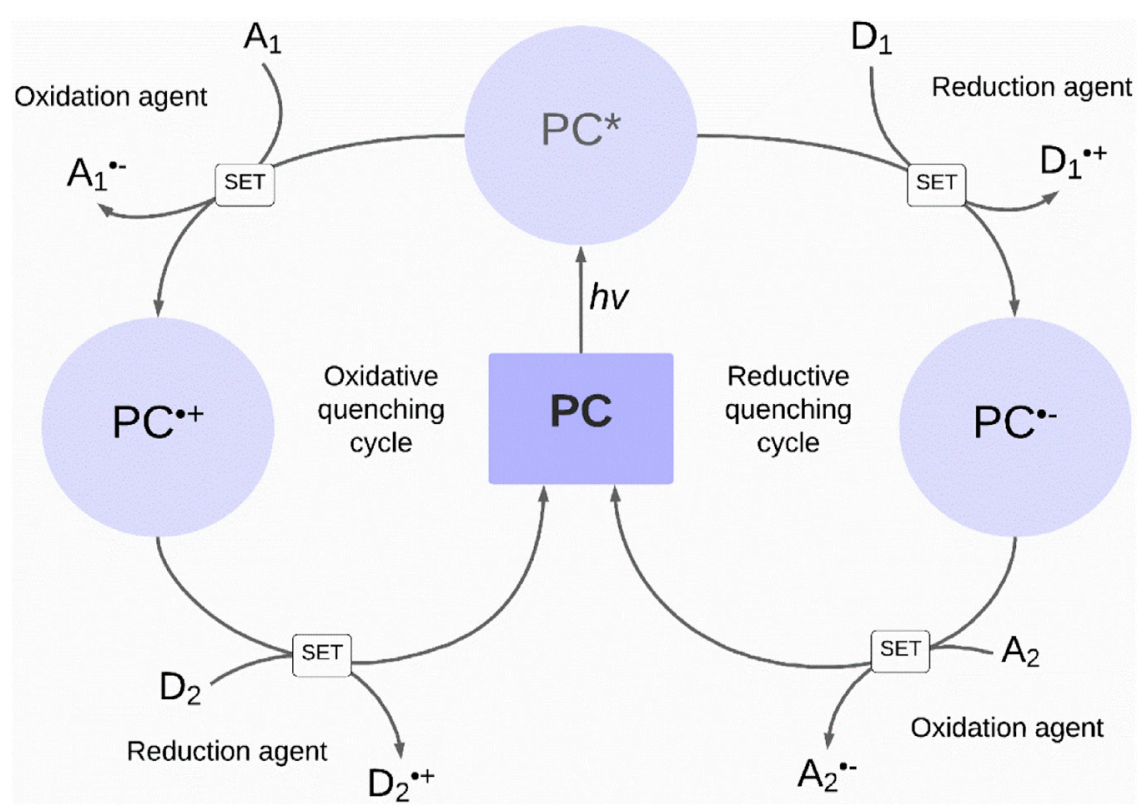

FIGURE 1 | Oxidative and reducing quenching cycle typical for a photoredox catalyst.

Furthermore, prediction of the effect of different operating conditions and evaluation of different geometric configurations of the microfluidic device can also be estimated.

Thus, this article aims to assist researchers in the fundamentals of photoredox catalysis and CFD-based technology for simulation of the reaction environment in photoredox catalysis, from the compilation of data in the literature as research gaps were identified on this theme, justifying further investigation.

\section{PHOTOREDOX CATALYSIS: FUNDAMENTALS AND APPLICATION IN THE SYNTHESIS OF HIGH ADDED-VALUE CHEMICALS}

Photoredox catalysis is a branch of catalysis that uses light energy to accelerate a chemical reaction through simple electron transfer events. As the same name implies, "photo" refers to light and "redox" is an expression that represents the chemical processes of reduction and oxidation.

In photoredox catalysis, the absorption of a photon by the photoredox catalyst in the visible or UV region is necessary. The catalyst excited by light allows electron transfer processes to occur with the agents, and oxidation and reduction reactions may be possible. When the photoredox catalyst is excited, an electron moves from the HOMO orbital (abbreviation of the highest occupied molecular orbital) to the LUMO orbital (abbreviation of the lowest unoccupied molecular orbital). As a result, there is a lack of an electron in HOMO and an electron available in LUMO (Anslyn and Dougherty, 2006; Romero and Nicewicz, 2016; Bonardi et al., 2018). That is why the excited photoredox catalyst is both a stronger oxidizer and a stronger reducer than its ground state. Therefore, the photocatalyst (PC) can easily react with an external oxidizer or reducer. Most of the photoredox catalytic reactions follow the catalytic cycle described in Figure 1.

Following light absorption, the photoexcited species perform the redox reactions in two different ways, depending on the reaction conditions employed and the properties of the sensitizer (Corrigan et al., 2016). As illustrated, the excited photocatalyst $\left(\mathrm{PC}^{\star}\right)$ reacts first with an oxidizing agent $\left(\mathrm{A}_{1}\right.$, electron acceptor), which leads to $\mathrm{PC}^{\bullet+}$ and $\mathrm{A}_{1}{ }^{\bullet-}$. Then, the $\mathrm{PC}$ can be regenerated by a reduction reaction with a reducing agent $\left(\mathrm{D}_{2}\right.$, electron donor), featuring the oxidative quenching cycle. On the other hand, in a reductive cycle, the $\mathrm{PC}^{\star}$ reacts first with the reducing agent $\left(\mathrm{D}_{1}\right)$ which leads to the $\mathrm{PC}^{\circ-}$ and $\mathrm{D}_{1}{ }^{\bullet+}$. Again, the $P C$ can be regenerated from a reaction with an oxidizing agent $\left(\mathrm{A}_{2}\right)$ (Koike and Akita, 2014; Romero and Nicewicz, 2016; Bonardi et al., 2018). Such a single electron transfer (SET) events have led to a better radical generation and give new life to a radical transformation in synthesis. The terms reductive and oxidative can be confusing and require explanations. Oxidative means oxidation of the photo-excited species $\mathrm{PC}^{\star}$ concomitant with reduction of the external electron acceptor A. Reductive refers to the reduction of photo-excited species $\mathrm{PC}^{*}$, while the external electron donor $\mathrm{D}$ is oxidized in the same process. In either case, by adding external redox agents, the photocatalyst can be regenerated.

Despite a large number of studies on photoredox catalysis, a very small set of photoredox catalysts are employed. They can be represented by transition metals complexes or by organic dyes capable of absorbing visible light, resulting in a kind of excited state. Among the catalyzed reactions based on transition metals, they typically employ metallic polypyridyl complexes, such as $\mathrm{Ru}$ (II) polypyridine complexes and cyclometalated Ir (III) complexes, 
TABLE 1 | Summary of the application of photoredox catalysis for the synthesis of added-value chemicals.

\begin{tabular}{|c|c|c|c|}
\hline Objective & Approach & Contribution & References \\
\hline $\begin{array}{l}\text { Develop a simple and direct method of } \\
\text { trifluoromethylation and perfluoroalkylation }\end{array}$ & $\begin{array}{l}\text { Visible light-mediated photoredox catalysis in a } \\
\text { continuous flow microreactor }\end{array}$ & $\begin{array}{l}\text { The methodology was significantly accelerated } \\
\text { (hours to minutes when converting from batch to } \\
\text { continuous flow system) }\end{array}$ & $\begin{array}{l}\text { Straathof et al. } \\
\text { (2014) }\end{array}$ \\
\hline Develop a hydroaminomethylation of olefins & $\begin{array}{l}\text { Photoredox catalysis with Boc-protected } \\
\text { aminomethyltrifluoroborate (Boc = tert- } \\
\text { butoxycarbonyl) }\end{array}$ & $\begin{array}{l}\text { The protocol provided a new strategy for the } \\
\text { synthesis of biologically active derivatives of } \\
\gamma \text {-aminobutyric acid (GABA), such as baclofen }\end{array}$ & $\begin{array}{l}\text { Miyazawa et al. } \\
\text { (2014) }\end{array}$ \\
\hline $\begin{array}{l}\text { Perform a direct application of carboxylic acids as } \\
\text { traceless activation group without screening for the } \\
\text { addition of radical conjugates }\end{array}$ & Visible light-mediated photoredox catalysis & $\begin{array}{l}\text { The approach presented itself adaptable to a } \\
\text { wide range of functional groups and has a wide } \\
\text { range of applications for both the carboxylic acid } \\
\text { and Michael acceptor components }\end{array}$ & $\begin{array}{l}\text { Chu et al. } \\
(2014)\end{array}$ \\
\hline $\begin{array}{l}\text { Realize the direct methylation of } \mathrm{C}-\mathrm{H} \text { in the late- } \\
\text { stage, ethylation and cyclopropanation of } \\
\text { pharmaceutical and agrochemicals }\end{array}$ & Visible light-mediated photoredox catalysis & $\begin{array}{l}\text { The procedure obtained high yields and the } \\
\text { photoredox catalysis was able to activate organic } \\
\text { peroxides to suitable radical alkylating agents }\end{array}$ & $\begin{array}{l}\text { DiRocco et al. } \\
\text { (2014) }\end{array}$ \\
\hline $\begin{array}{l}\text { Develop the dehydrogenation for the synthesis of } \\
\text { elbasvir, an inhibitor of the hepatitis } C \text { virus }\end{array}$ & Visible light-mediated photoredox catalysis & $\begin{array}{l}\text { The approach was able to produce the product } \\
\text { in high quantities while maintaining optical purity }\end{array}$ & $\begin{array}{l}\text { Yayla et al. } \\
(2016)\end{array}$ \\
\hline $\begin{array}{l}\text { Demonstrate hydrogen atom transfer protocol to } \\
\text { install deuterium and tritium efficiently and } \\
\text { selectively at a-amino sp3 carbon-hydrogen bonds } \\
\text { in a single step }\end{array}$ & Photoredox catalysis & $\begin{array}{l}\text { The authors successfully applied this protocol to } \\
18 \text { drug molecules. The same research group } \\
\text { from MacMillan developed studies in the field of } \\
\text { metallaphotoredox, allowing the rapid and } \\
\text { efficient synthesis of medicinal agents, such as } \\
\text { the synthesis of fenofibrate (Chu et al., 2015; Gu } \\
\text { et al., 2016) and the synthesis of edivoxetine (Le } \\
\text { and MacMillan, 2015) }\end{array}$ & $\begin{array}{l}\text { Loh et al. } \\
(2017)\end{array}$ \\
\hline $\begin{array}{l}\text { Obtain tertiary alkyl fluorides from methyl oxalates } \\
\text { derived from tertiary alcohols }\end{array}$ & $\begin{array}{l}\text { Sequential one-pot hydrolysis/photoredox } \\
\text { catalyzed radical deoxyfluorination sequence }\end{array}$ & $\begin{array}{l}\text { The simple production of tertiary alkyl fluorides } \\
\text { under photoredox catalysis at room temperature } \\
\text { is a useful addition to the published methods }\end{array}$ & Brioche (2018) \\
\hline Demonstrate the difunctionalization of ethylene & Photoredox catalysis & $\begin{array}{l}\text { Ethylene performed the divergent synthesis of } \\
\text { 1,2-diarylananes, 1,4-diarylbutanes or } 2,3- \\
\text { diarylbutanes in a highly selective way }\end{array}$ & Li et al. (2019) \\
\hline $\begin{array}{l}\text { Develop the organophotocatalytic direct } \\
\text { difluoromethylation of heterocycles using } \mathrm{O}_{2} \text { as a } \\
\text { green oxidizer }\end{array}$ & Photoredox catalysis & $\begin{array}{l}\text { This methodology demonstrates the feasibility of } \\
\text { late drug development. The authors assessed } \\
\text { the bioactivity of the synthesis product in which it } \\
\text { had promising activity against cancer cell lines }\end{array}$ & $\begin{array}{l}\text { Zhang et al. } \\
(2020)\end{array}$ \\
\hline
\end{tabular}

offering charge transfer properties for metal binders (Koike and Akita, 2014; Wang et al., 2018; Glaser and Wenger, 2020). On the other hand, metal-free conditions have been developed using organic dyes such as Eosin, Rhodamine, rose Bengal, 9fluorenone, xanthone, methylene blue, acridinium, etc., capable of absorbing light in or near the visible region (Nicewicz and Nguyen, 2014; Wang et al., 2018; Zhou et al., 2019).

For a compound to be efficient as a photoredox catalyst, there are some desirable characteristics (Arias-Rotondo and Mccusker, 2016; Romero and Nicewicz, 2016):

1) Strong light absorption on the spectral region of the agents of interest;

2) Stability in the solution;

3) The lifetime of the excited state long enough to react with the agents;

4) The oxidation or reduction of the photoredox catalyst must be reversible to be regenerated;

5) Reversible redox behavior so that the photoredox catalyst is regenerated (to avoid any side reaction from $\mathrm{PC}^{\bullet+}$ and $\mathrm{PC}^{\bullet-}$ ).
These catalysts were successfully implemented for the discovery of new organic reactions and the synthesis of added-value chemicals, with excellent control of selectivity and stereoregularity, creating a new approach to organic transformations.

In recent years, the field of photoredox catalysis has experienced exponential growth, resulting in the development of a wide variety of new synthesis methodologies (Shaw et al., 2016; McAtee et al., 2019). In this scenario, the successful application of processes mediated by visible light to pharmaceutical products and biologically relevant molecules is essential for its continued expansion. Here, we will discuss some applications of the utility of photoredox catalysis in the synthesis of added-value chemicals. Table 1 presents a simplified summary of works reported in the literature for photoredox catalysis in the synthesis of added-value chemicals.

As a result, it is evident that the photoredox catalysis has become a powerful research area for chemical synthesis, with an increasing number of publications. This technology has found applications in a variety of synthetically useful reactions of organic compounds, even using metallic complexes or organic dyes as catalysts. 


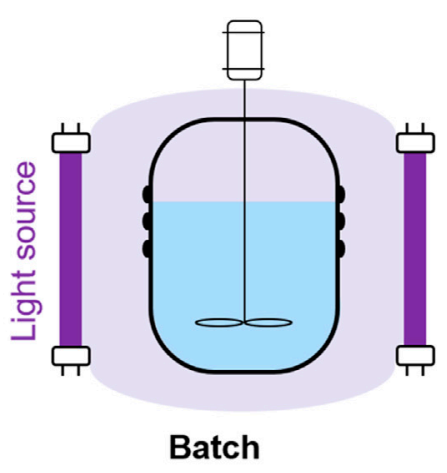

- Nonuniform irradiation

- Limited reaction volumes

- Batch-to-batch variations

- Inefficient mass and heat transfer

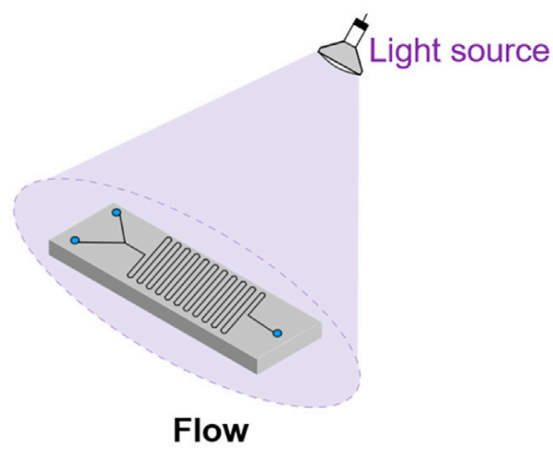

- Uniform irradiation

- Unlimited reaction volumes

- Enhanced reproducibility

- Very fast mixing

- Short residence times

- No concentration/temperature gradients

FIGURE 2 | Schematic representation and comparisons of batch and flow systems.

\section{BATCH VERSUS FLOW PHOTOCHEMISTRY}

Photochemical reactions occur when light provides energy to trigger a reaction (Plutschack et al., 2017). It includes electron transfer chemistry initiated by the excitation of a chromophore (photoredox catalysis), an attractive method for organic synthesis. Thus, it is evident that photochemistry, in general, is heavily dependent on the efficient irradiation of the reaction mixture. There are currently two main types of reactor systems that researchers use for photochemistry: batch and flow (Figure 2).

Continuous flow (micro) reactors have been highlighted as a technology of choice for photochemical reactions to overcome the bottleneck of dependence on efficient irradiation of the reaction mixture (Cambié et al., 2016; Reischauer and Pieber, 2021). The main benefits of continuous-flow microreactors over batch reactors are high surface-to-volume ratio and small scale, favorable characteristics for improving mass and photon transfer (Van Gerven et al., 2007; Lira et al., 2021). In addition, other advantages are short molecular diffusion distances, laminar flow, and fast and efficient heat dissipation (Vesborg et al., 2010; Padoin et al., 2016; Heggo and Ookawara, 2017; Yusuf et al., 2018). However, to achieve higher reaction rates, the distance required for the interaction between the components must be shorter. The smaller dimensions of the channels favor the decrease of sample volumes, which leads to a reduction in the amount of reagents used for tests and analyzes (Nge et al., 2013). Therefore, recent advances in this field mean better efficiency in terms of kinetics, safety and cost (Wang et al., 2002; Plutschack et al., 2017). Because of these advantages, the synthesis of high added-value compounds in continuous flow microreactors has become a promising area in recent years (Wiles and Watts, 2012; Rashmi Pradhan et al., 2019).

Additionally, when it comes to light-dependent systems (photochemical reactions), the use of continuous microreactors leads to improved irradiation on the reaction mixture due to better light penetration throughout the reactor depth and greater homogeneity of space lighting (Matsushita et al., 2007; Krivec et al., 2013). As revealed by the well-known Beer-LambertBouguer law, the radiation distribution will not be uniform in a reactor due to absorption effects (Cambié et al., 2016). This equation establishes the light absorption $(\boldsymbol{A})$ is dependent on the molecule's molar attenuation coefficient $(\boldsymbol{\varepsilon})$, their concentration $(\boldsymbol{c})$, and the path length of the light propagation $(\boldsymbol{l})$ (Eq. 1).

$$
\boldsymbol{A}=\log _{10} \boldsymbol{T}=\log _{10} \frac{\boldsymbol{I}_{0}}{\boldsymbol{I}}=\boldsymbol{\varepsilon} \boldsymbol{c l} .
$$

Unfortunately for batch reactors, this compensation has serious implications, especially for sizing a photochemical reaction. The decrease in radiation intensity with distance from the light source in batch reactors becomes one of the main disadvantages for photochemistry (Knowles et al., 2012; Oelgemoeller, 2012). In addition to avoiding this disadvantage of conventional reactors, microfluidic devices are also attractive for catalytic reactions due to their large surface-to-volume ratio and the excellent control over the reaction conditions they offer. Consequently, photochemical reactions in continuous flow reactors can be substantially accelerated and scaled to larger quantities (Reischauer and Pieber, 2021).

Cambié et al. (2016) reported two important reasons for photochemistry to reach so much repercussion in academic and industrial areas. The first one is the exposure of visible photo-redox catalysis to organic synthetic chemistry. The second reason is the use of continuous flow reactors. Additionally, the same authors list nine good reasons for using photoflow: 1) better irradiation of the reaction mixture; 2) reliable increase; 3 ) better reaction selectivity and greater reproducibility; 4) fast mixing; 5) rapid heat exchange; 6) multiphase chemistry; 7) reaction sequences in several stages; 8) immobilized catalysts and 9) greater operational safety. 
Noël et al. (2011) compared the results of the microfluidic system with those obtained in batch experiments. The authors noted that there was no loss of activity when performed inflow. In fact, in the flow reaction, they observed greater activity in very short residence times. This result demonstrated one of the main advantages of flow chemistry. The authors stated that the increase in heat and mass transfer allowed the microreactor to heat more quickly and efficiently. Therefore, these properties make it ideal for quick reactions. Similarly, Nair et al. (2019) demonstrated that continuous-flow reactors have a competent configuration compared to batch reactors due to the high conversion rates and selectivity for the selective organic synthesis of aromatic alcohols. In addition, Abdiaj and Alcázar (Abdiaj and Alcázar, 2017) demonstrated an improved efficacy for photoredox and nickel catalysis for $\mathrm{C}\left(\mathrm{sp}^{2}\right)-\mathrm{C}\left(\mathrm{sp}^{3}\right)$ cross-coupling using a continuous-flow reactor instead of a batch system. The authors mentioned that the space-time yield was 430 times better than the batch method, thus resulting in a more suitable procedure for drug discovery in terms of productivity per unit of time. Interestingly, Oelgemöller and Shvydkiv (Oelgemöller and Shvydkiv, 2011) summarized advances in microflow photochemical transformations. The authors presented comparative details between the batch system vs. microreactor for several examples of homogeneous and heterogeneous reactions, including photoadditions, photorearrangements, photoreductions, photodecarboxylations, photooxygenations and photochlorinations. In general, microfluidic devices have resulted in reduced irradiation times, greater selectivity and greater light efficiency. Therefore, the microreactors demonstrated their superiority over conventional techniques.

Although, in theory, the use of flow reactors for photochemical reactions has numerous benefits, as shown in this section, compared to batch reactors; however, developing a flow process can be time-consuming and there are still several limitations. One of the biggest limitations of flow chemistry is the handling of solids (Gisbertz and Pieber, 2020). Plutschack et al. (2017) developed a very interesting decision diagram to try to facilitate decision-making for flow versus batch analysis with the aim of finding the balance between convenience and achieving the overall goal. Therefore, it is expected that new equipment concepts applied to organic synthesis are expected to emerge as an important future area of research and development (R\&D) and production methodology to improve synthetic efficiency through process automation and optimization.

\section{MODELING AND SIMULATION OF PHOTOREDOX CATALYTIC PROCESSES IN FLOW REACTOR}

As technologies become more developed and commercialized, they can help and speed up research based on experimental laboratory tests (Plutschack et al., 2017). Simulation and numerical modeling have become a more widely used tool to gain deeper insights into the system and to examine features that are difficult in experiments
(Dong et al., 2021). Computational methods can be employed in the prediction and design of photochemical reactions, and also it allows scientists to gain deeper physical insights that are not easily accessible experimentally (Lira et al., 2021). Thus, computational fluid dynamics (CFD) has been widely and consistently used in reactive systems modeling. This development was made possible by significant advances in computing power and numerical models due to the high dimensionality, complexity, and highly non-linear dependencies on the properties and responses of continuous-flow devices. This area requires a greater computational effort, but even so, it shows promise in reducing the time of analysis and optimization of the photochemical reaction.

Photoredox catalytic processes in continuous-flow reactors are significantly more technologically complex, i.e., not as trivial as traditional batch techniques, in which the reactants are simply added into a vessel and stirred at a defined temperature until that the limiting reagent is consumed (Plutschack et al., 2017). Therefore, the limitations of photon and mass transfer can be addressed through the development of mathematical models that characterize the effect of fluid dynamics on photochemical reactions and represent the transport of light through the flow reactor. Thus, a set of partial nonlinear differential equations must be solved for a complete description of coupling, the fluid flow, mass transport, and photon transport (irradiation distribution) for photoredox catalytic processes in continuous flow reactors (Lira et al., 2021). The three-dimensional governing equations for continuity, momentum, and mass conservation of chemical species in Cartesian coordinates can be given by Eqs 2-4, respectively, according to Bird et al. (2007).

$$
\begin{gathered}
\frac{\partial \rho}{\partial t}+\nabla \cdot(\rho \mathbf{v})=0, \\
\rho \frac{D}{D t} \mathbf{v}=-\nabla p+\mu \nabla^{2} \mathbf{v}+\rho \mathbf{g}, \\
\frac{\partial C_{i}}{\partial t}=-\nabla \cdot\left(\mathbf{v} C_{i}\right)+\nabla \cdot\left(D_{i} \nabla C_{i}\right)+r_{i}\left(\left\{C_{i}\right\}\right),
\end{gathered}
$$

where, $\rho$ is the density of the mixture, $\mathrm{v}$ is the velocity vector, $p$ is the pressure, $\mu$ is the dynamic viscosity of the mixture, $\rho \mathrm{g}$ is the gravitational force, $C_{i}$ is the concentration of species $i, D_{i}$ is the diffusion coefficient of the species $i$ and $r_{i}\left(\left\{C_{i}\right\}\right)$ term represents the consumption or production rate of species $i$.

However, the main challenge for reaction engineering in photoredox catalysis processes is that, in addition to all the parameters that govern a catalytic reaction, the photocatalyst must first be activated by light. The photons distribution becomes more problematic with increasing reactor dimensions because they have a non-homogeneous light distribution. This scenario presents a non-trivial problem in modeling and optimizing the reaction parameters since the reaction rate must take into account the local volumetric rate of photon absorption (Bloh and Marschall, 2017), as the problems associated with the radioactive and chemical reaction are well interconnected. In a photoreaction, these two problems are coupled and the solution of the governing equations (mass balance of reactive species and radiative transfer) ends up being a very complex problem (Martin et al., 1999). Besides that, in the last decades, the study of 
photochemical processes has mainly involved research for the formation of new products, reaction kinetics and mechanisms; however, the implementation of the energy source is often neglected (Su et al., 2014). This is the main reason why very little is known today about how photon transport influences photoredox catalysis and opens new horizons for the development of studies, modeling, simulation and optimization for this type of reaction.

\subsection{Radiation Transport Modeling}

As mentioned earlier, one of the greatest challenges related to continuous-flow photochemistry is the participation of radiation in chemical reactions. Thus, the conversion and reaction rates are critically dependent on the efficiency of the radiation field. The lack of homogeneity of the irradiation and the corresponding variations in the reaction conditions can reduce the yield and selectivity. Therefore, in this section, we will highlight the radiation transport modeling, and later, we will list the main types of light sources that can be used in photochemical processes.

Currently, many studies report photochemical processes, either for the synthesis of added-value chemicals or for photodegradation; however, few of them are the studies that evaluate the effect of photon transport (Visan et al., 2014; Van Gerven et al., 2007). This can be solved by associating simulation software with experimental tests of chemical transformations in microreactors. Rigorous modeling of the radiation field in photochemical processes requires solving the radiation transport equation (RTE) in the appropriate coordinate system. This equation can be associated with the reactive flow inside microfluidic devices from CFD codes. The general form of the RTE can be written according to Eq. 5, and it describes the movement of the beam of light rays traveling in the $s$ direction and having a solid angle $\Omega$, intensity $I$, and wavelength $\lambda$ (Loubière et al., 2016; Nicholls et al., 2016).

$$
\begin{aligned}
\frac{d I_{\lambda}(s, \boldsymbol{\Omega})}{d s}=- & \kappa_{a, \lambda} I_{\lambda}(s, \boldsymbol{\Omega})-\sigma_{s, \lambda} I_{\lambda}(s, \boldsymbol{\Omega}) \\
& +\frac{\sigma_{s, \lambda}}{4 \pi} \int_{0}^{4 \pi} p\left(\Omega^{\prime} \rightarrow \Omega\right) I_{\lambda}\left(s, \Omega^{\prime}\right) d \Omega^{\prime},
\end{aligned}
$$

where, $\boldsymbol{\kappa}_{\boldsymbol{a}, \lambda}$ means the volumetric absorption coefficient, $\boldsymbol{\sigma}_{\boldsymbol{s}, \lambda}$ is the volumetric scattering coefficient, and $p\left(\Omega^{\prime} \rightarrow \Omega\right)$ is the phase function that describes the directional distribution of scattered radiation.

As RTE is an integral-differential equation, its analytical solutions are only possible under specific conditions; for most practical reactors, numerical methods should be used (Boyjoo et al., 2013). Different approaches for the RTE numerical solution have been developed and used in recent years to describe the radiant energy field in photochemistry processes. Examples are analytical approaches such as the Two- and Six-Flux Model (TFM and SFM, respectively) (Li Puma and Brucato, 2007; RamírezCabrera et al., 2017), Monte Carlo (MC) stochastic method (Zazueta et al., 2013; Akach and Ochieng, 2018), discrete ordinates method (DOM) (Boyjoo et al., 2014), among others.

The two-flux model (TFM) and the six-flux model (SFM) were developed for analytical approximations to the RTE solution in an annular slurry photoreactor (Li Puma et al., 2004; Li Puma and Brucato, 2007). The TFM model assumes that the photons travel in two directions (forward and backwards) and the SFM model represents the external dispersion in the six directions of the Cartesian coordinates. The advantage of these models is their ability to estimate the local volumetric rate photon absorption (LVRPA) using simple algebraic equations (Peralta Muniz Moreira and Li Puma, 2021). Different configurations were also used to describe the radiation field using mathematical models, such as multi-annular reactor (Imoberdorf et al., 2007) and corrugated plate reactor (Passalía et al., 2011). However, no work was found using these radiation field models for application in micro (photo) reactors.

The P1 approximation to solving the RTE, also called the differential approximation, is the most general case for solving the radioactive problem (Arancibia-Bulnes and Cuevas, 2004; Cuevas et al., 2007; Boyjoo et al., 2013). This model assumes that the intensity field depends only weakly on the direction. A linear dependence is assumed, which transforms the RTE into a second degree linear partial differential equation (Ramírez-Cabrera et al., 2017). The P1 model can be solved for simpler cases, obtaining satisfactory results with low absorption coefficients and zerodispersion; however, it has also been shown that the same model can be very imprecise for high absorption coefficients (Yu et al., 2008).

Another fact that requires further consideration is that photons are not entirely directed to the reaction medium after impinging the system, which directly contributes to the system's irradiation efficiency. In this sense, Monte Carlo method can be used to solve the RTE solution, probabilistically simulating the possible trajectory of photons in a medium, including absorption, scattering and reflection (Boyjoo et al., 2013; Asadollahfardi et al., 2018; Ayoub, 2020; Akach et al., 2020). This method involves statistical sampling to model complex chemical and physical phenomena and has shown good results when applied to model the radiation field in photochemistry systems (Singh et al., 2007). Cambié et al. (2017) used Monte Carlo raytracing simulations for a detailed description of the photon path within the LSC-PM microdevice [Figure 3 (i-a)]. The relative contributions of the different photon fates were obtained, generating spectral distribution of the photons per each fate, resulting in a complete photon-balance graph [Figure 3 (i-b and c)]. It was found that the largest fraction in the photon balance, $43 \%$ of the photons reaching the device, is constituted by the transmitted photons. These simulation results were then used to investigate the expected efficiency of the extended devices. The authors noted that it would be possible to obtain accurate information about the instantaneous flow of photons reaching the reaction channel just by monitoring the variation in the photons emitted by the edges. Matiazzo et al. (2022) explored the impact of the LEDs-to-LEDs distance and also the evaluation of the LEDs-reactor distance from a nonsequential ray tracer based on Monte Carlo. Therefore, it is worth noting that the great advantage of algorithms based on Monte Carlo is the ability to deal with complex geometries without excessive computational demands. 

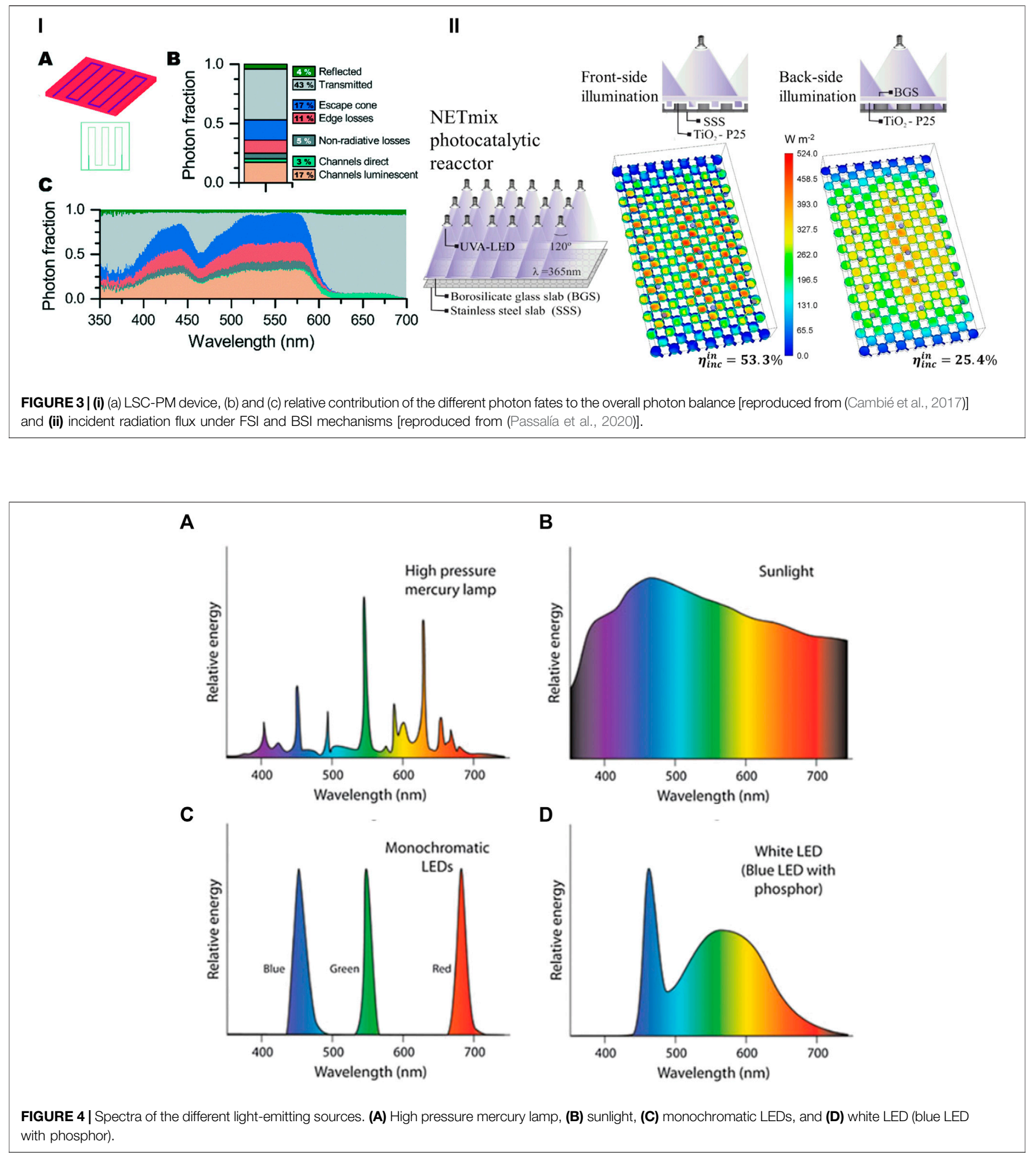

The discrete ordinates method (DOM) consists of transforming the RTE differential-integral form into a system of algebraic equations that can be solved by a computer (Jensen et al., 2007). This system of equations describes the transport of photons in a way that can be solved following the direction of propagation, starting from the values provided by the boundary conditions (Alvarado-Rolon et al., 2018). This method solves the RTE for a finite number of discrete solid angles, each associated with a vector direction (Casado et al., 2017a). Currently, the DOM model is used by CFD software to model the radiation field and can be coupled with the reaction kinetics. The 
ability of this model to provide more accurate results for problems with a radiation field coupled with a chemical reaction has made it widely applied for photochemical application (Coelho, 2014; Alvarado-Rolon et al., 2018; Moreno et al., 2019; Lira et al., 2020; Peralta Muniz Moreira and Li Puma, 2021). For example, Passalía et al. (2020) simulated an array of 18 LEDs to light a milli-reactor known as NETmix employing a DOM method, and they used the oxidation of trivalent arsenate to pentavalent arsenate in aqueous solutions as a reaction model [Figure 3 (ii)]. Two lighting settings were compared: front-side (FSI) and back-side (BSI) illumination. And therefore, from the radiation modeling, the results showed that although the FSI configuration presents a larger surface area, this configuration is worse or less illuminated since most of the catalytic surface corresponds to the side walls. Similarly, Casado et al. (2017b) used DOM to solve the RTE, allowing the evaluation of the radiation field at any point inside the reactor to evaluate and optimize the irradiation distribution with LEDs.

\subsubsection{Types of Radiation Sources}

One of the most important factors in the study of photochemical processes is the choice of the light source. Some considerations must be made while choosing a light source. The optimal wavelength for the process must be determined, and this stage includes in most of the times a UV/vis characterization of the reaction substrate or photosensitizer (Williams and Kappe, 2020). The spectra distribution of some of the most used light sources can be seen in Figure 4.

Another aspect that needs attention is the overlap between the emission spectrum of the light source and the absorption spectrum of absorbent species or photoactive catalysts. Moreover, for photochemical synthesis, the choice of a suitable light source depends on the volume of synthesis and the intensity of light required. However, for safety reasons, protection needs to be considered to prevent damage from the light source and the ozone generated if UV light is used (Liu, 2017). Lamps for photochemical applications are characterized by their spectral purity and radiant emission power. The physical principle behind all radiation sources is the luminescence of excited atoms or molecules, in which the electronic transition from excited states to ground state leads to the emission of radiation (Pareek et al., 2008). Based on this, light sources can be divided into the following categories: mercury discharge lamps, light-emitting diodes (LEDs) and sunlight.

\section{- Mercury discharge lamps}

Currently, the most popular light sources are mercury lamps due to their convenience because they are easy to use and provide light with wavelengths ranging from ultraviolet to visible $(200-750 \mathrm{~nm})$ (Figure 4A). These light sources are usually located outside the microreactors, and the capillary version is usually wrapped around the lamps (Su et al., 2014). The incorporation of these light sources inside the microreactors is not possible since the dimensions of these lamps are much larger than the microreactors.

In mercury gas discharge lamps, the light originates from an excited electron in the mercury atom, returning to its ground state energy level and emitting radiation (Kuipers, 2014). This light source has a long life, has a relatively efficient light emission and can emit over a wide wavelength range. Based on the mercury vapor pressure in the lamps, they can be classified as low pressure $(0.6665-13.33 \mathrm{~Pa})$, medium pressure $\left(1.013 \times 10^{4}-1.013 \times\right.$ $\left.10^{5} \mathrm{~Pa}\right)$ and high pressure $\left(2.026 \times 10^{7} \mathrm{~Pa}\right)$ (Liu, 2017). Regarding use, medium and low-pressure lamps are often used for medium and low UV wavelengths (UV-A and UV-B, $350-250 \mathrm{~nm})$, while high lamps emit a length from where up to $600 \mathrm{~nm}$ (Cambié et al., 2016).

Despite the advantages associated with this type of lamp, they have some disadvantages, of which one of the most pressing is the use of mercury. The use of mercury is severely restricted due to its high toxicity to humans and the environment.

\section{- Solar light}

The use of sunlight as a light source for chemical transformations is motivated, in part, by the perception that sunlight is effectively a source of clean and inexhaustible energy (Hoffmann, 2008). Sunlight and other polychromatic light sources are used due to their universality, as it is possible to choose wavelengths suitable for the study process (Figure $4 \mathbf{B}$ ). However, in photochemical transformations, most organic molecules absorb only ultraviolet (UV) light, and they cannot be activated by the visible wavelengths that comprise most of the solar energy that reaches the Earth's surface (Schultz and Yoon, 2014). Incompatible light energy can allow unwanted reaction pathways, leading to substantial by-product formation. Another disadvantage of this type of light source is attributed to the low and transient intensity of solar radiation and its diffuse nature (dispersion by clouds, buildings, etc.), significantly reducing the performance of photochemical processes (Cambié et al., 2016).

- Light-emitting diodes (LEDs)

Recently, light-emitting diodes (LEDs) have become more popular for microfluidic applications; however their narrow wavelength range may limit their use $( \pm 20 \mathrm{~nm}$ ) ( $\mathrm{Su}$ et al., 2014) (Figures 4C,D). The LEDs are semiconductors (PN junction) capable of emitting light when activated, due to the electroluminescence produced via recombination of electrons and gaps. The wavelength emitted by this type of light source can be adapted (from UV to Vis light) and combined with the photochemical application (Cambié et al., 2016). With LEDs, it is possible to produce artificial light with selected peak wavelength emission that closely matches the absorption peak of a known important photoreceptor (Dutta Gupta et al., 2017).

The advantages of LEDs are high energy efficiency, small size, low cost, durability, long life and can be made of non-toxic materials, different from mercury discharge lamps. With these advantages, this type of light source is ideal for combining with continuous flow reactors. Additionally, the LEDs have low energy loss, and only a certain amount of heat is generated, i.e., practically all electrical energy can be transformed into light monochrome energy (Preto et al., 2018). A disadvantage of LEDs is their light emission as a point source and the intensity of a single LED is quite low; however, this problem can be reversed by combining several LEDs to form a matrix, thus providing efficient irradiation (Sender-E et al., 2017). 
I (a) [4+2] cycloaddition using photogenerated singlet oxygen<smiles>c1ccc(-c2c3ccccc3c(-c3ccccc3)c3ccccc23)cc1</smiles>
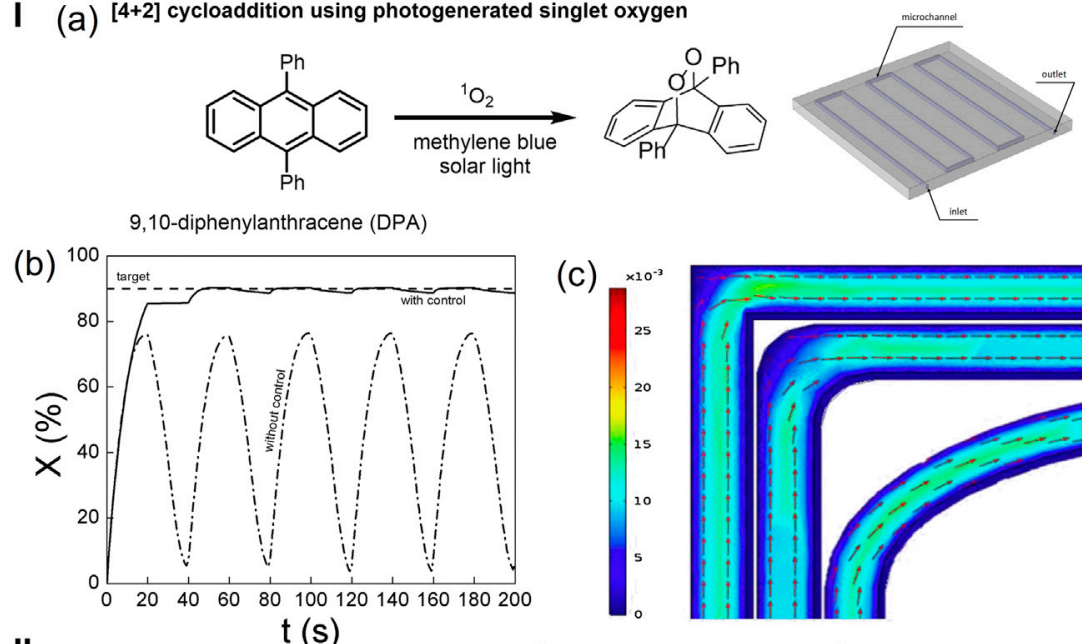

(c)

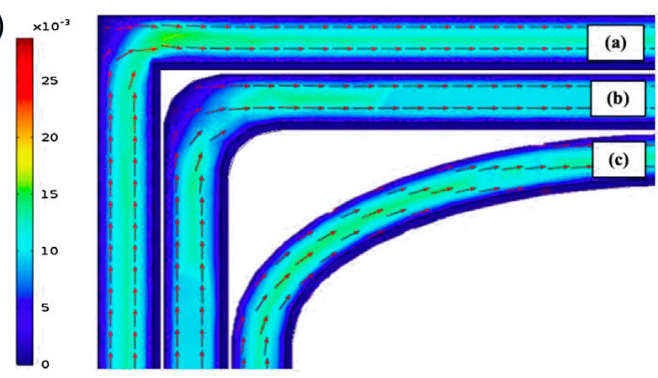

II (a)

$\mathrm{t}(\mathrm{s})$
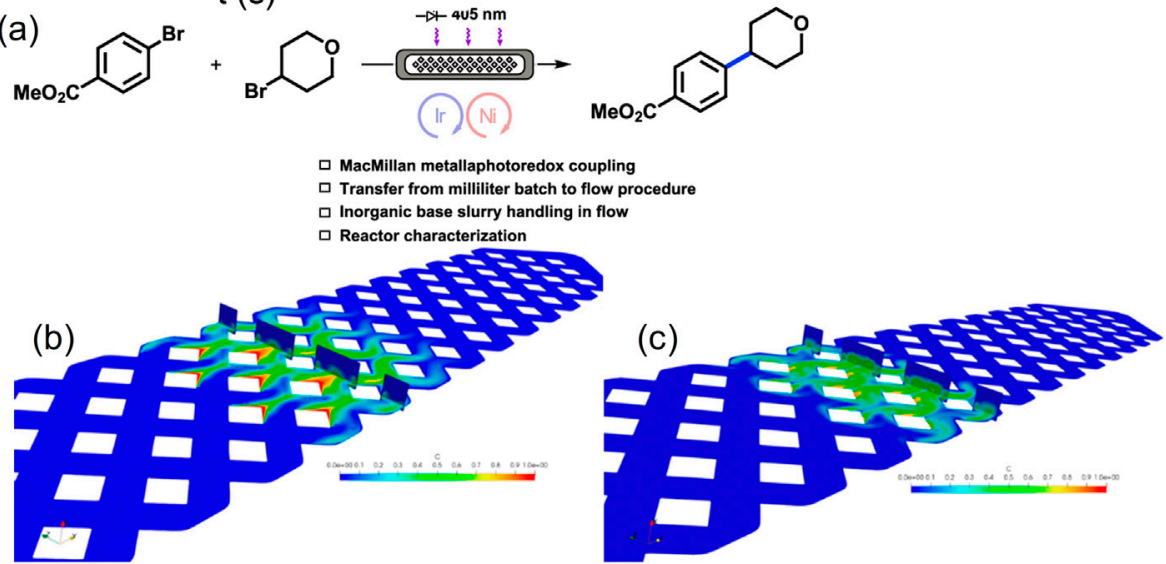

FIGURE 5 | (i) (a) [4 + 2] cycloaddition of 9,10-diphenylanthracene (DPA) using photogenerated singlet oxygen, (b) process with and without the control system during the power disturbances, and (c) non-ideal flow effects on velocity in three different channel configurations [reproduced from (de Oliveira et al., 2020)]. (ii) (a) Ir/Ni Photoredox $\mathrm{C}\left(\mathrm{sp}^{2}\right)-\mathrm{C}\left(\mathrm{sp}^{3}\right)$ Cross-Electrophile Coupling of Methyl 4-Bromobenzoate and 4-Bromotetrahydropyran in the continuous flow regime, concentration field in the reactor $\mathrm{t}=1.0 \mathrm{~s}$ after tracer injection with (b) no pulsation and (c) with pulsation frequency of $3 \mathrm{~Hz}$ and amplitude of $33 \%$ [reproduced from (Debrouwer et al., 2020)].

\subsection{Computational Fluid Dynamics Towards an Optimized Photoredox Catalysis Process Design}

As progress continues for the photoredox catalysis, there is a constant need for a deeper understanding of how the process for refining chemical methods takes place to enable improvements in this technology. This is due to the fact that a photoredox catalysis is mainly an experimental approach, and, therefore, the method only offers an overview and does not allow a detailed study inside the reactor. In particular, a complete understanding of hydrodynamics, chemical reaction, and the influence of light in the photoredox catalysis process would be of paramount importance for the design of microreactors and optimization of the added-value chemicals synthesis. Therefore, the numerical modeling of these processes can be utilized in conjunction with the experimental component to assess the viability of the process and reach higher degrees of precision. Thus, computational simulation (CFD) becomes essential for the design and optimization of microfluidic devices applied to the photoredox catalysis, as it avoids the costly time and money expenses, in addition to providing increasingly efficient microdevices (Padoin et al., 2014; Padoin and Soares, 2017; Lira et al., 2019; Lira et al., 2020; Cao et al., 2022).

CFD stands out as a useful tool as it considers the coupling of all phenomena that occur (fluid flow, mass transport, and photon transport). Therefore, once the CFD-based model has been validated, investigations of velocity profiles, radiation and concentration distribution, prediction of the effect of different operating conditions, and geometric configurations can be easily performed with virtual prototypes (Mohajerani et al., 2012). Currently, several commercials software are used to solve the numerical equations that describe the microreactor systems. Examples of such software are ANSYS Fluent ${ }^{\circledR}$, COMSOL Multiphysics ${ }^{\circledR}$, OpenFOAM ${ }^{\circledR}$, among others. Despite the vast number of software available on the market, the basic principle is the same for everyone, where the CFD code is divided into three 
main steps: pre-processing, solver and post-processing. With this, it is possible to evaluate fluid flows, evaluate the influence of channel geometry only with virtual prototypes and optimize the micro-reactive system (Santana et al., 2020).

It is worth noting that many studies have been carried out to simulate CFD-based micro-flow devices. Environmental protection, power plants, and added-value chemicals can be listed among the various sectors in which this technology has already been implemented, performing single-phase or multiphase flows (Cao et al., 2022). An et al. (An et al., 2012) examined eight different configurations of a microreactor in the form of a square cross-section channel via computational fluid dynamics to determine which results in the best performance. Similarly, Odiba et al. (Odiba and Olea, 2016) explored the behavior of the fluid in the microchannels of four different geometries of a microreactor from CFD simulation applied in the catalytic oxidation of volatile organic compounds. Ortega-Casanova (Ortega-Casanova, 2017) applied CFD in the optimization process of a chemical microreactor for the mixing efficiency evaluation. On the other hand, Alfano and Cassano (Alfano and Cassano, 2009) developed a general procedure to increase the scale of photoreactors of any type using modeling with the mass balances, at the moment, of radiation with the kinetic model.

However, when it comes to photoredox catalysis, few works have been reported in the literature involving CFD simulations. de Oliveira et al. (2020) implemented a CFD-based virtual model of the luminescent solar concentrator-based photomicroreactor (LSC-PM). The CFD model was validated with the experimental data based on the $[4+2]$ cycloaddition reaction of 9,10-diphenylanthracene, and in the CFD code, a feedforward control system was implemented [Figure 5 (i-a and b)]. Furthermore, an investigation of the geometric characteristic of the microchannel was carried out, highlighting the ability to use the computational model for design purposes [Figure 5 (i-c)]. The formation of dead zones in the square-channel microreactor was verified when analyzing the flow effect for the analyzed geometries. It corroborated with the decrease in conversion for this channel setting compared to the curved channel. Debrouwer et al. (2020) used CFD simulation to support the beneficial effect of pulsation on the residence time distribution for MacMillan's light-driven $\mathrm{C}\left(\mathrm{sp}^{2}\right)-\mathrm{C}\left(\mathrm{sp}^{3}\right)$ cross-electrophile coupling [Figure 5 (ii-a)]. Moreover, by calculating tracer transport over time, these simulations allow comparison of vertical mixing times in the absence and presence of pulsation based on the concentration field in the reactor [Figure 5 (ii-b and c)]. It was found that the time required for the species to be transported to half the channel height is less than $1 \mathrm{~s}$ when the pulse is applied. Aillet et al. (2015) used the CFD code to predict the performance (conversion and photonic efficiency) of an $A \rightarrow{ }^{h v} B$ photochemical reaction, where A and B compete for the absorption of incident photons. Interestingly, the model of this work (Aillet et al., 2015) is expressed using classical dimensionless numbers such as Damköhler numbers I and II, absorbance, and competitive absorption factor. Differently, although Sezen-Edmonds et al. (2020) did not use CFD, they demonstrated a modeling tool to predict the performance of a photochemical reaction as a function of easily measurable parameters. Experimental data were combined with computational modeling for two case studies: 1) [2+2] photocycloaddition reaction and 2) decarboxylative intramolecular arene alkylation mediated by photoredox catalysis.

It is worth noting that, in addition to CFD-based simulations, some authors are using ray-tracing modeling to visualize photon transport to optimize the design of reactors applied to photochemical reactions (Buglioni et al., 2021). It can be realized using a Monte Carlo ray-trace algorithm from the code development (Zhu et al., 2020) or through the use of commercial software such as LightTools ${ }^{\circledR}$ (Vishwanathan et al., 2015), Ray Optics Module (an add-on to COMSOL Multiphysics ${ }^{\circledR}$ ) (Jacobs et al., 2020; Zheng et al., 2020), ANSYS SPEOS ${ }^{\circledR}$ (Matiazzo et al., 2022), among others.

Thus, the use of computational software, whether CFD or based on a ray-tracing algorithm, is essential for the development and improvement of reactors for photochemical reactions where there is a coupling of hydrodynamics, mass transport, and photon transport. However, more studies still need to be conducted and published for photoredox catalysis reactions so that researchers understand more deeply the phenomena that occur in this type of reaction. Therefore, the modeling and simulation of reaction environments in photoredox catalysis still has a lot to be explored to establish itself as a reliable technology.

\section{DISCUSSIONS ON FUTURE DIRECTIONS AND CONCLUDING REMARKS}

Photoredox catalysis has been a transforming force in synthetic organic chemistry in the last decade, mainly for the pharmaceutical sector. This means that academic discoveries have a better chance of being promptly converted into future technology. There are no doubt that the photoredox catalysis processes will continue to use (micro) flow reactors to perform organic photochemical reactions. This growing popularity of the microreactors can be attributed to the high yields (in terms of space/time), high selectivity, and few side reactions (when compared to batch reactors) achieved by the majority of these reactors.

However, as the field of photoredox catalysis advances, a better understanding of how the process of refining chemical techniques works is required to enable advancements in this technology. For the design of microreactors and optimization of the synthesis of added-value compounds, a comprehensive understanding of the principles between the reagents, as well as a perception of the influence of light on the photoredox catalysis process, would be crucial. Nevertheless, the challenge is to integrate the complexity of continuous flow photochemical processes in order to elucidate some gaps found when approaching this subject.

For that, computational tools can provide, in the research and development of microfluidic devices for photoredox catalysis, an immediate qualitative and quantitative analysis of the species' behavior and light distribution. Furthermore, knowledge of fluid flows is also essential to adequate the design and optimization of micro(photo)reactors. The great advantages of following the computational path are the decrease in the timeconsuming and money expenses and the possibility of providing 
increasingly efficient models. In this sense, CFD can stand out as a powerful tool for solving and analyzing transport equations. However, difficulties linked to light efficiency, such as dissipation, penetration, and transmission of light, are still not taken into account in those CFD simulations. Other computational methods capable of simulating this phenomenon are being sought as a solution to this problem. Therefore, the framework consisting of the combination of photon fate modeling and CFD can be crucial to improve the performance of the photoredox catalysis process.

In this present literature review, we summarized the advances made in photoredox catalysis, highlighting the computational numerical approach. It was noteworthy that great efforts have been made to improve the understanding of the fundamentals and application of this technology for the added-value chemicals synthesis. Furthermore, the advantages of working with a continuous flow system were also understood. Despite efforts, photoredox catalysis is still a challenging issue as it involves the consideration of several simultaneous phenomena. Therefore, computer simulation of these

\section{REFERENCES}

Abdiaj, I., and Alcázar, J. (2017). Improving the Throughput of Batch Photochemical Reactions Using Flow: Dual Photoredox and Nickel Catalysis in Flow for C(sp2)C(sp3) Cross-Coupling. Bioorg. Med. Chem. 25, 6190-6196. doi:10.1016/J.BMC.2016.12.041

Aillet, T., Loubiere, K., Dechy-Cabaret, O., and Prat, L. (2013). Photochemical Synthesis of a "Cage" Compound in a Microreactor: Rigorous Comparison with a Batch Photoreactor. Chem. Eng. Process. Process Intensification 64, 38-47. doi:10.1016/J.CEP.2012.10.017

Aillet, T., Loubière, K., Prat, L., and Dechy-Cabaret, O. (2015). Impact of the Diffusion Limitation in Microphotoreactors. Aiche J. 61, 1284-1299. doi:10.1002/aic.14718

Akach, J., Kabuba, J., and Ochieng, A. (2020). Simulation of the Light Distribution in a Solar Photocatalytic Bubble Column Reactor Using the Monte Carlo Method. Ind. Eng. Chem. Res. 59, 17708-17719. doi:10.1021/ acs.iecr.0c02124

Akach, J., and Ochieng, A. (2018). Monte Carlo Simulation of the Light Distribution in an Annular Slurry Bubble Column Photocatalytic Reactor. Chem. Eng. Res. Des. 129, 248-258. doi:10.1016/j.cherd.2017.11.021

Alfano, O. M., and Cassano, A. E. (2009). Scaling-Up of Photoreactors. Applications to Advanced Oxidation Processes. Adv. Chem. Eng. 36, 229-287. doi:10.1016/S0065-2377(09)00407-4

Alvarado-Rolon, O., Natividad, R., Romero, R., Hurtado, L., and Ramírez-Serrano, A. (2018). Modelling and Simulation of the Radiant Field in an Annular Heterogeneous Photoreactor Using a Four-Flux Model. Int. J. Photoenergy 2018, 1-16. doi:10.1155/2018/1678385

An, H., Li, A., Sasmito, A. P., Kurnia, J. C., Jangam, S. V., and Mujumdar, A. S. (2012). Computational Fluid Dynamics (CFD) Analysis of Micro-reactor Performance: Effect of Various Configurations. Chem. Eng. Sci. 75, 85-95. doi:10.1016/j.ces.2012.03.004

Anslyn, E. V., and Dougherty, D. A. (2006). "Photochemistry," in Mod. Phys. Org. Chem. (Sausalito, CA, United States: University Science Books), 1095.

Arancibia-Bulnes, C. A., and Cuevas, S. A. (2004). Modeling of the Radiation Field in a Parabolic Trough Solar Photocatalytic Reactor. Solar Energy 76, 615-622. doi:10.1016/j.solener.2003.12.001

Arias-Rotondo, D. M., and Mccusker, J. K. (2016). The Photophysics of Photoredox Catalysis: a Roadmap for Catalyst Design. Chem. Soc. Rev. 45, 5803-5820. doi: $10.1039 / \mathrm{c} 6 \mathrm{cs} 00526 \mathrm{~h}$

Armstrong, C. T., Pritchard, C. Q., Cook, D. W., Ibrahim, M., Desai, B. K., Whitham, P. J., et al. (2019). Continuous Flow Synthesis of a Pharmaceutical reaction systems is a scientific hotspot for researchers to clarify improves the interaction between reagents and the influence of light on the reaction medium. However, the simulation of photoredox catalysis for the added-value chemicals synthesis still has a lot to be explored to establish itself as a reliable approach.

\section{AUTHOR CONTRIBUTIONS}

All authors listed have made a substantial, direct, and intellectual contribution to the work and approved it for publication.

\section{ACKNOWLEDGMENTS}

The authors acknowledge CAPES (Coordination for the Improvement of Higher-Level Personnel and CNPq (National Council for Scientific and Technological Development) for the financial support.

Intermediate: A Computational Fluid Dynamics Approach. React. Chem. Eng. 4, 634-642. doi:10.1039/c8re00252e

Asadollahfardi, G., Noori, M., Asadi, M., and Taherioun, M. (2018). The Comparison of Discrete Ordinate and Monte Carlo Methods in Solving of the Radiation Transfer Equations in a Heterogenous Reactor. J. Water Supply Res. Tec 67, 109-118. doi:10.2166/aqua.2017.058

Ayoub, M. (2020). A Review on Light Transport Algorithms and Simulation Tools to Model Daylighting inside Buildings. Solar Energy 198, 623-642. doi:10.1016/ j.solener.2020.02.018

Bird, R. B., Stewart, W. E., and Lightfoot, E. N. (2007). Transport Phenomena. 2nd ed. Hoboken, New Jersey, United States: John Wiley \& Sons.

Bloh, J. Z., and Marschall, R. (2017). Heterogeneous Photoredox Catalysis: Reactions, Materials, and Reaction Engineering. Eur. J. Org. Chem. 2017, 2085-2094. doi:10.1002/ejoc.201601591

Bonardi, A.-H., Dumur, F., Noirbent, G., Lalevée, J., and Gigmes, D. (2018). Organometallic vs Organic Photoredox Catalysts for Photocuring Reactions in the Visible Region. Beilstein J. Org. Chem. 14, 3025-3046. doi:10.3762/ bjoc. 14.282

Boyjoo, Y., Ang, M., and Pareek, V. (2014). CFD Simulation of a Pilot Scale Slurry Photocatalytic Reactor and Design of Multiple-Lamp Reactors. Chem. Eng. Sci. 111, 266-277. doi:10.1016/j.ces.2014.02.022

Boyjoo, Y., Ang, M., and Pareek, V. (2013). Some Aspects of Photocatalytic Reactor Modeling Using Computational Fluid Dynamics. Chem. Eng. Sci. 101, 764-784. doi:10.1016/j.ces.2013.06.035

Brioche, J. (2018). One-pot Synthesis of Tertiary Alkyl Fluorides from Methyl Oxalates by Radical Deoxyfluorination under Photoredox Catalysis. Tetrahedron Lett. 59, 4387-4391. doi:10.1016/J.TETLET.2018.10.063

Buglioni, L., Raymenants, F., Slattery, A., Zondag, S. D. A., and Noël, T. (2021). Technological Innovations in Photochemistry for Organic Synthesis: Flow Chemistry, High-Throughput Experimentation, Scale-Up, and Photoelectrochemistry. Chem. Rev. doi:10.1021/acs.chemrev.1c00332

Cambié, D., Bottecchia, C., Straathof, N. J. W., Hessel, V., and Noël, T. (2016). Applications of Continuous-Flow Photochemistry in Organic Synthesis, Material Science, and Water Treatment. Chem. Rev. 116, 10276-10341. doi:10.1021/acs.chemrev.5b00707

Cambié, D., Zhao, F., Hessel, V., Debije, M. G., and Noël, T. (2017). Every Photon Counts: Understanding and Optimizing Photon Paths in Luminescent Solar Concentrator-Based Photomicroreactors (LSC-PMs). React. Chem. Eng. 2, 561-566. doi:10.1039/c7re00077d

Cao, Y., Padoin, N., Soares, C., and Noël, T. (2022). On the Performance of LiquidLiquid Taylor Flow Electrochemistry in a Microreactor - A CFD Study. Chem. Eng. J. 427, 131443. doi:10.1016/j.cej.2021.131443 
Casado, C., Marugán, J., Timmers, R., Muñoz, M., and van Grieken, R. (2017). Comprehensive Multiphysics Modeling of Photocatalytic Processes by Computational Fluid Dynamics Based on Intrinsic Kinetic Parameters Determined in a Differential Photoreactor. Chem. Eng. J. 310, 368-380. doi:10.1016/j.cej.2016.07.081

Casado, C., Timmers, R., Sergejevs, A., Clarke, C. T., Allsopp, D. W. E., Bowen, C. R., et al. (2017). Design and Validation of a LED-Based High Intensity Photocatalytic Reactor for Quantifying Activity Measurements. Chem. Eng. J. 327, 1043-1055. doi:10.1016/J.CEJ.2017.06.167

Chu, L., Lipshultz, J. M., and Macmillan, D. W. C. (2015). Merging Photoredox and Nickel Catalysis: The Direct Synthesis of Ketones by the Decarboxylative Arylation of a-Oxo Acids. Angew. Chem. Int. Ed. 54, 7929-7933. doi:10.1002/anie.201501908

Chu, L., Ohta, C., Zuo, Z., and MacMillan, D. W. C. (2014). Carboxylic Acids as A Traceless Activation Group for Conjugate Additions: A Three-step Synthesis of (士)-Pregabalin. J. Am. Chem. Soc. 136, 10886-10889. doi:10.1021/ja505964r

Coelho, P. J. (2014). Advances in the Discrete Ordinates and Finite Volume Methods for the Solution of Radiative Heat Transfer Problems in Participating media. J. Quantitative Spectrosc. Radiative Transfer 145, 121-146. doi:10.1016/ j.jqsrt.2014.04.021

Corrigan, N., Shanmugam, S., Xu, J., and Boyer, C. (2016). Photocatalysis in Organic and Polymer Synthesis. Chem. Soc. Rev. 45, 6165-6212. doi:10.1039/ c6cs00185h

Coyle, E. E., and Oelgemöller, M. (2008). Micro-photochemistry: Photochemistry in Microstructured Reactors. The New Photochemistry of the Future? Photochem. Photobiol. Sci. 7, 1313-1322. doi:10.1039/b808778d

Crisenza, G. E. M., and Melchiorre, P. (2020). Chemistry Glows green with Photoredox Catalysis. Nat. Commun. 11, 8-11. doi:10.1038/s41467-01913887-8

Cuevas, S. A., Arancibia-Bulnes, C. A., and Serrano, B. (2007). Radiation Field in an Annular Photocatalytic Reactor by the P1 Approximation. Int. J. Chem. React. Eng. 5. doi:10.2202/1542-6580.1589

Lira, J. O. de. B., Padoin, N., Vilar, V. J. P., and Soares, C. (2019). Photocatalytic NOx Abatement: Mathematical Modeling, CFD Validation and Reactor Analysis. J. Hazard. Mater. 372, 145-153. doi:10.1016/ j.jhazmat.2018.07.009

de Oliveira, G. X., Lira, J. O. B., Cambié, D., Noël, T., Riella, H. G., Padoin, N., et al. (2020). CFD Analysis of a Luminescent Solar ConcentratorBased Photomicroreactor (LSC-PM) with Feedforward Control Applied to the Synthesis of Chemicals under Fluctuating Light Intensity. Chem. Eng. Res. Des. 153, 626-634. doi:10.1016/j.cherd.2019.10.047

Debrouwer, W., Kimpe, W., Dangreau, R., Huvaere, K., Gemoets, H. P. L., Mottaghi, M., et al. (2020). Ir/Ni Photoredox Dual Catalysis with Heterogeneous Base Enabled by an Oscillatory Plug Flow Photoreactor. Org. Process. Res. Dev. 24, 2319-2325. doi:10.1021/acs.oprd.0c00150

DiRocco, D. A., Dykstra, K., Krska, S., Vachal, P., Conway, D. V., and Tudge, M. (2014). Late-stage Functionalization of Biologically Active Heterocycles through Photoredox Catalysis. Angew. Chem. Int. Ed. 53, 4802-4806. doi:10.1002/anie.201402023

Dong, Z., Wen, Z., Zhao, F., Kuhn, S., and Noël, T. (2021). Scale-up of Micro- and Milli-Reactors: An Overview of Strategies, Design Principles and Applications. Chem. Eng. Sci. X 10, 100097. doi:10.1016/j.cesx.2021.100097

Dutta Gupta, S., and Agarwal, A. (2017). “Artificial Lighting System for Plant Growth and Development: Chronological Advancement, Working Principles, and Comparative Assessment," in Light Emit. Diodes Agric. Smart Light. Editor S. D. Gupta (Singapore: Springer Nature Singapore Pte Ltd), 1-25. doi:10.1007/978-981-10-5807-3_1

Gisbertz, S., and Pieber, B. (2020). Heterogeneous Photocatalysis in Organic Synthesis. ChemPhotoChem 4, 456-475. doi:10.1002/cptc.202000014

Glaser, F., and Wenger, O. S. (2020). Recent Progress in the Development of Transition-Metal Based Photoredox Catalysts. Coord. Chem. Rev. 405, 213129. doi:10.1016/J.CCR.2019.213129

Gu, L., Jin, C., Liu, J., Zhang, H., Yuan, M., and Li, G. (2016). Acylation of Indoles via Photoredox Catalysis: A Route to 3-acylindoles. Green. Chem. 18, 1201-1205. doi:10.1039/c5gc01931a

Heggo, D., and Ookawara, S. (2017). Multiphase Photocatalytic Microreactors. Chem. Eng. Sci. 169, 67-77. doi:10.1016/j.ces.2017.01.019

Hoffmann, N. (2008). Photochemical Reactions as Key Steps in Organic Synthesis. Chem. Rev. 108, 1052-1103. doi:10.1021/cr0680336
Imoberdorf, G. E., Cassano, A. E., Irazoqui, H. A., and Alfano, O. M. (2007). Simulation of a Multi-Annular Photocatalytic Reactor for Degradation of Perchloroethylene in Air: Parametric Analysis of Radiative Energy Efficiencies. Chem. Eng. Sci. 62, 1138-1154. doi:10.1016/j.ces.2006.10.024

Jacobs, M., Kayahan, E., Thomassen, L. C. J., Kuhn, S., and Leblebici, M. E. (2020). First Generation of Translucent Monoliths for Photochemical Applications. Jnl Adv. Manuf Process. 2, 1-11. doi:10.1002/amp2.10047

Jensen, K. F., Reizman, B. J., and Newman, S. G. (2014). Tools for Chemical Synthesis in Microsystems. Lab. Chip 14, 3206-3212. doi:10.1039/ c4lc00330f

Jensen, K., Ripoll, J., Wray, A., Joseph, D., and Elhafi, M. (2007). On Various Modeling Approaches to Radiative Heat Transfer in Pool Fires. Combustion and Flame 148, 263-279. doi:10.1016/j.combustflame.2006.09.008

Knowles, J. P., Elliott, L. D., and Booker-Milburn, K. I. (2012). Flow Photochemistry: Old Light through New Windows. Beilstein J. Org. Chem. 8, 2025-2052. doi:10.3762/bjoc.8.229

Koike, T., and Akita, M. (2014). Visible-light Radical Reaction Designed by $\mathrm{Ru}$ - and Ir-Based Photoredox Catalysis. Inorg. Chem. Front. 1, 562-576. doi:10.1039/C4QI00053f

Krivec, M., Žagar, K., Suhadolnik, L., Čeh, M., and Dražić, G. (2013). Highly Efficient TiO2-Based Microreactor for Photocatalytic Applications. ACS Appl. Mater. Inter. 5, 9088-9094. doi:10.1021/am402389t

Kuipers, J. (2014). "Distributed Light Sources for Photocatalytic Water Treatment," (Wageningen, NL: Thesis Wageningen University). Thesis.

Le, C. C., and MacMillan, D. W. C. (2015). Fragment Couplings via CO2 Extrusion-Recombination: Expansion of a Classic Bond-Forming Strategy via Metallaphotoredox. J. Am. Chem. Soc. 137, 11938-11941. doi: $10.1021 /$ jacs.5b08304

Li, J., Luo, Y., Cheo, H. W., Lan, Y., and Wu, J. (2019). Photoredox-CatalysisModulated, Nickel-Catalyzed Divergent Difunctionalization of Ethylene. Chem 5, 192-203. doi:10.1016/J.CHEMPR.2018.10.006

Li Puma, G., and Brucato, A. (2007). Dimensionless Analysis of Slurry Photocatalytic Reactors Using Two-Flux and Six-Flux Radiation Absorption-Scattering Models. Catal. Today 122, 78-90. doi:10.1016/ j.cattod.2007.01.027

Li Puma, G., Khor, J. N., and Brucato, A. (2004). Modeling of an Annular Photocatalytic Reactor for Water Purification: Oxidation of Pesticides. Environ. Sci. Technol. 38, 3737-3745. doi:10.1021/es0301020

Lira, J. O. B., Riella, H. G., Padoin, N., and Soares, C. (2020). CFD + DoE Optimization of a Flat Plate Photocatalytic Reactor Applied to NOx Abatement. Chem. Eng. Process. - Process Intensification 154, 107998. doi:10.1016/ j.cep.2020.107998

Lira, J. O. de. B., Riella, H. G., Padoin, N., and Soares, C. (2021). An Overview of Photoreactors and Computational Modeling for the Intensification of Photocatalytic Processes in the Gas-phase : State-Of-Art. J. Environ. Chem. Eng. 9, 105068. doi:10.1016/j.jece.2021.105068

Liu, X.-S. (2017). Inorganic Photochemical Synthesis. Iselin, NJ, United States: Elsevier B.V., 143-165. doi:10.1016/B978-0-444-63591-4.00006-9

Loh, Y. Y., Nagao, K., Hoover, A. J., Hesk, D., Rivera, N. R., Colletti, S. L., et al. (2017). Photoredox-catalyzed Deuteration and Tritiation of Pharmaceutical Compounds. Science 358, 1182-1187. doi:10.1126/science.aap9674

Loubière, K., Oelgemöller, M., Aillet, T., Dechy-Cabaret, O., and Prat, L. (2016). Continuous-flow Photochemistry: A Need for Chemical Engineering. Chem. Eng. Process. Process Intensification 104, 120-132. doi:10.1016/ J.CEP.2016.02.008

Martin, C. A., Camera-Roda, G., and Santarelli, F. (1999). Effective Design of Photocatalytic Reactors: Influence of Radiative Transfer on Their Performance. Catal. Today 48, 307-313. doi:10.1016/S0920-5861(98)00386-1

Matiazzo, T., Ramaswamy, K., Vilar, V. J. P., Padoin, N., and Soares, C. (2022). Radiation Field Modeling of the NETmix Milli-Photocatalytic Reactor: Effect of LEDs Position over the Reactor Window. Chem. Eng. J. 429, 131670. doi:10.1016/j.cej.2021.131670

Matsushita, Y., Ichimura, T., Ohba, N., Kumada, S., Sakeda, K., Suzuki, T., et al. (2007). Recent Progress on Photoreactions in Microreactors. Pure Appl. Chem. 79, 1959-1968. doi:10.1351/pac200779111959

McAtee, R. C., McClain, E. J., and Stephenson, C. R. J. (2019). Illuminating Photoredox Catalysis. Trends Chem. 1, 111-125. doi:10.1016/ J.TRECHM.2019.01.008 
Miyazawa, K., Koike, T., and Akita, M. (2014). Hydroaminomethylation of Olefins with Aminomethyltrifluoroborate by Photoredox Catalysis. Adv. Synth. Catal. 356, 2749-2755. doi:10.1002/adsc.201400556

Mohajerani, M., Mehrvar, M., and Ein-Mozaffari, F. (2012). "Computational Fluid Dynamics (CFD) Modeling of Photochemical Reactors," in Applied Computational Fluid Dynamics. Editor H. W. Oh (IntechOpen), 154-176. doi:10.5772/26468

Moreno, J., Casado, C., and Marugán, J. (2019). Improved Discrete Ordinate Method for Accurate Simulation Radiation Transport Using Solar and LED Light Sources. Chem. Eng. Sci. 205, 151-164. doi:10.1016/ j.ces.2019.04.034

Nair, V., Colmenares, J. C., and Lisovytskiy, D. (2019). Ultrasound Assisted ZnO Coating in a Microflow Based Photoreactor for Selective Oxidation of Benzyl Alcohol to Benzaldehyde. Green. Chem. 21, 1241-1246. doi:10.1039/c8gc03131b

Narayanam, J. M. R., and Stephenson, C. R. J. (2011). Visible Light Photoredox Catalysis: Applications in Organic Synthesis. Chem. Soc. Rev. 40, 102-113. doi:10.1039/b913880n

Nge, P. N., Rogers, C. I., and Woolley, A. T. (2013). Advances in Microfluidic Materials, Functions, Integration, and Applications. Chem. Rev. 113, 2550-2583. doi:10.1021/ cr300337x

Nicewicz, D. A., and Nguyen, T. M. (2014). Recent Applications of Organic Dyes as Photoredox Catalysts in Organic Synthesis. ACS Catal. 4, 355-360. doi:10.1021/ $\operatorname{cs} 400956 \mathrm{a}$

Nicholls, T. P., Leonori, D., and Bissember, A. C. (2016). Applications of Visible Light Photoredox Catalysis to the Synthesis of Natural Products and Related Compounds. Nat. Prod. Rep. 33, 1248-1254. doi:10.1039/c6np00070c

Noël, T. (2017). A Personal Perspective on the Future of Flow Photochemistry. J. Flow Chem. 7, 87-93. doi:10.1556/1846.2017.00022

Noël, T., Naber, J. R., Hartman, R. L., Mcmullen, J. P., Jensen, K. F., and Buchwald, S. L. (2011). Palladium-catalyzed Amination Reactions in Flow: Overcoming the Challenges of Clogging via Acoustic Irradiation. Chem. Sci. 2, 287-290. doi: $10.1039 / \mathrm{c} 0 \mathrm{sc} 00524 \mathrm{j}$

Oelgemoeller, M. (2012). Highlights of Photochemical Reactions in Microflow Reactors. Chem. Eng. Technol. 35, 1144-1152. doi:10.1002/ ceat.201200009

Oelgemöller, M., and Shvydkiv, O. (2011). Recent Advances in Microflow Photochemistry. Molecules 16, 7522-7550. doi:10.3390/molecules16097522

Oliveira de Brito Lira, J., Riella, H. G., Padoin, N., and Soares, C. (2021). An Overview of Photoreactors and Computational Modeling for the Intensification of Photocatalytic Processes in the Gas-phase: State-Of-Art. J. Environ. Chem. Eng. 9, 105068. doi:10.1016/j.jece.2021.105068

Ortega-Casanova, J. (2017). Application of CFD on the Optimization by Response Surface Methodology of a Micromixing Unit and its Use as a Chemical Microreactor. Chem. Eng. Process. Process Intensification 117, 18-26. doi:10.1016/J.CEP.2017.03.012

Padoin, N., Andrade, L., Ângelo, J., Mendes, A., Moreira, R. d. F. P. M., and Soares, C. (2016). Intensification of Photocatalytic Pollutant Abatement in Microchannel Reactor Using TiO2and TiO2-Graphene. Aiche J. 62, 2794-2802. doi:10.1002/aic10.1002/aic.15262

Padoin, N., Dal'Toé, A. T. O., Rangel, L. P., Ropelato, K., and Soares, C. (2014). Heat and Mass Transfer Modeling for Multicomponent Multiphase Flow with CFD. Int. J. Heat Mass Transfer 73, 239-249. doi:10.1016/ j.ijheatmasstransfer.2014.01.075

Padoin, N., and Soares, C. (2017). An Explicit Correlation for Optimal TiO 2 Film Thickness in Immobilized Photocatalytic Reaction Systems. Chem. Eng. J. 310, 381-388. doi:10.1016/j.cej.2016.06.013

Pareek, V., Chong, S., Tadé, M., and Adesina, A. A. (2008). Light Intensity Distribution in Heterogenous Photocatalytic Reactors. Asia-pacific Jrnl Chem. Eng. 3, 171-201. doi:10.1002/apj10.1002/apj.129

Passalía, C., Alfano, O. M., Brandi, R. J., Passalía, C., Alfano, O. M., Brandi, R. J., et al. (2011). Modeling and Experimental Verification of a Corrugated Plate Photocatalytic Reactor Using Computational Fluid Dynamics. Ind. Eng. Chem. Res. 50, 9077-9086. doi:10.1021/ie200756t

Passalía, C., Flores, M., Santos, S. G. S., Paulista, L. O., Labas, M. D., Vilar, V. J. P., et al. (2020). Radiation Modelling in the NETmix Photocatalytic Reactor: The Concept of Efficiencies in Series. J. Environ. Chem. Eng. 8, 104464. doi:10.1016/ j.jece.2020.104464

Peralta Muniz Moreira, R., and Li Puma, G. (2021). Multiphysics Computational Fluid-Dynamics (CFD) Modeling of Annular Photocatalytic Reactors by the
Discrete Ordinates Method (DOM) and the Six-Flux Model (SFM) and Evaluation of the Contaminant Intrinsic Kinetics Constants. Catal. Today 361, 77-84. doi:10.1016/J.CATTOD.2020.01.012

Plutschack, M. B., Pieber, B., Gilmore, K., and Seeberger, P. H. (2017). The Hitchhiker's Guide to Flow Chemistry. Chem. Rev. 117, 11796-11893. doi:10.1021/acs.chemrev.7b00183

Preto, S., and Gomes, C. C. (2018). "Ultraviolet Radiation in Sunlight and Artificial Lighting Systems: Are They Alike" in Adv. Intell. Syst. Comput. Editors R. Goonetilleke and W. Karwowski (Cham: Springer), 407-416. doi:10.1007/ 978-3-319-60825-9

Ramírez-Cabrera, M. A., Valadés-Pelayo, P. J., Arancibia-Bulnes, C. A., and Ramos, E. (2017). Validity of the Six-Flux Model for Photoreactors. Chem. Eng. J. 330, 272-280. doi:10.1016/J.CEJ.2017.07.120

Rashmi Pradhan, S., Colmenares-Quintero, R. F., and Colmenares Quintero, J. C. (2019). Designing Microflowreactors for Photocatalysis Using Sonochemistry: A Systematic Review Article. Molecules 24, 3315. doi:10.3390/molecules24183315

Reischauer, S., and Pieber, B. (2021). Emerging Concepts in Photocatalytic Organic Synthesis. IScience 24, 102209-102217. doi:10.1016/j.isci.2021.102209

Romero, N. A., and Nicewicz, D. A. (2016). Organic Photoredox Catalysis. Chem. Rev. 116, 10075-10166. doi:10.1021/acs.chemrev.6b00057

Odiba, S., and Olea, M., (2016). Computational Fluid Dynamics in Microreactors Analysis and Design: Application to Catalytic Oxidation of Volatile Organic Compounds. J. Chem. Eng. Process. Technol. 07, 297-308. doi:10.4172/2157-7048.1000297

Santana, H. S., da Silva, A. G. P., Lopes, M. G. M., Rodrigues, A. C., Taranto, O. P., and Lameu Silva, J. (2020). Computational Methodology for the Development of Microdevices and Microreactors with ANSYS CFX. MethodsX 7, 100765. doi:10.1016/J.MEX.2019.12.006

Schultz, D. M., and Yoon, T. P. (2014). Solar Synthesis: Prospects in Visible Light Photocatalysis. Science 343, 343. doi:10.1126/science.1239176

Sender- E, M. P. P., ZiegenbalgSender, D., and Ziegenbalg, D. (2017). Light Sources for Photochemical Processes - Estimation of Technological Potentials. Chem. Ingenieur Technik 89, 1159-1173. doi:10.1002/cite.201600191

Sezen-Edmonds, M., Tabora, J. E., Cohen, B. M., Zaretsky, S., Simmons, E. M., Sherwood, T. C., et al. (2020). Predicting Performance of Photochemical Transformations for Scaling up in Different Platforms by Combining HighThroughput Experimentation with Computational Modeling. Org. Process. Res. Dev. 24, 2128-2138. doi:10.1021/acs.oprd.0c00182

Shaw, M. H., Twilton, J., and MacMillan, D. W. C. (2016). Photoredox Catalysis in Organic Chemistry. J. Org. Chem. 81, 6898-6926. doi:10.1021/acs.joc.6b01449

Singh, M., Salvadó-Estivill, I., and Li Puma, G. (2007). Radiation Field Optimization in Photocatalytic Monolith Reactors for Air Treatment. Aiche J. 53, 678-686. doi:10.1002/aic10.1002/aic.11093

Straathof, N. J. W., Gemoets, H. P. L., Wang, X., Schouten, J. C., Hessel, V., and Noël, T. (2014). Rapid Trifluoromethylation and Perfluoroalkylation of Five-Membered Heterocycles by Photoredox Catalysis in Continuous Flow. ChemSusChem 7, 1612-1617. doi:10.1002/cssc.201301282

Straathof, N. J. W., Su, Y., Hessel, V., and Noël, T. (2016). Accelerated Gas-Liquid Visible Light Photoredox Catalysis with Continuous-Flow Photochemical Microreactors. Nat. Protoc. 11, 10-21. doi:10.1038/nprot.2015.113

Su, Y., Kuijpers, K., Hessel, V., and Noël, T. (2016). A Convenient NumberingUp Strategy for the Scale-Up of Gas-Liquid Photoredox Catalysis in Flow. React. Chem. Eng. 1, 73-81. doi:10.1039/c5re00021a

Su, Y., Straathof, N. J. W., Hessel, V., and Noël, T. (2014). Photochemical Transformations Accelerated in Continuous-Flow Reactors: Basic Concepts and Applications. Chem. Eur. J. 20, 10562-10589. doi:10.1002/ chem.201400283

Van Gerven, T., Mul, G., Moulijn, J., and Stankiewicz, A. (2007). A Review of Intensification of Photocatalytic Processes. Chem. Eng. Process. Process Intensification 46, 781-789. doi:10.1016/j.cep.2007.05.012

Vesborg, P. C. K., Olsen, J. L., Henriksen, T. R., Chorkendorff, I., and Hansen, O. (2010). Gas-phase Photocatalysis in $\mu$-reactors. Chem. Eng. J. 160, 738-741. doi:10.1016/j.cej.2010.03.083

Visan, A., Rafieian, D., Ogieglo, W., and Lammertink, R. G. H. (2014). Modeling Intrinsic Kinetics in Immobilized Photocatalytic Microreactors. Appl. Catal. B: Environ. 150-151, 93-100. doi:10.1016/j.apcatb.2013.12.003

Vishwanathan, B., Reinders, A. H. M. E., de Boer, D. K. G., Desmet, L., Ras, A. J. M., Zahn, F. H., et al. (2015). A Comparison of Performance of Flat and 
Bent Photovoltaic Luminescent Solar Concentrators. Solar Energy 112, 120-127. doi:10.1016/j.solener.2014.12.001

Wang, C.-S., Dixneuf, P. H., and Soulé, J.-F. (2018). Photoredox Catalysis for Building C-C Bonds from C(sp2)-H Bonds. Chem. Rev. 118, 7532-7585. doi:10.1021/acs.chemrev.8b00077

Wang, H., Nakamura, H., Uehara, M., Miyazaki, M., and Maeda, H. (2002). Preparation of Titania Particles Utilizing the Insoluble Phase Interface in a Microchannel Reactor. Chem. Commun. 14, 1462-1463. doi:10.1039/b203478f

Weissman, S. A., and Anderson, N. G. (2015). Design of Experiments (DoE) and Process Optimization. A Review of Recent Publications. Org. Process. Res. Dev. 19, 1605-1633. doi:10.1021/op500169m

Wiles, C., and Watts, P. (2012). Continuous Flow Reactors: a Perspective. Green. Chem. 14, 38-54. doi:10.1039/c1gc16022b

Williams, J. D., and Kappe, C. O. (2020). Recent Advances toward Sustainable Flow Photochemistry. Curr. Opin. Green Sustainable Chem. 25, 100351. doi:10.1016/j.cogsc.2020.05.001

Yayla, H. G., Peng, F., Mangion, I. K., McLaughlin, M., Campeau, L.-C., Davies, I. W., et al. (2016). Discovery and Mechanistic Study of a Photocatalytic Indoline Dehydrogenation for the Synthesis of Elbasvir. Chem. Sci. 7, 2066-2073. doi:10.1039/c5sc03350k

Yu, B., Deng, B., and Kim, C. N. (2008). Performance Evaluation of P-1 Model in a Photocatalytic Reactor. Chem. Eng. Sci. 63, 5552-5558. doi:10.1016/ j.ces.2008.08.002

Yusuf, A., Garlisi, C., and Palmisano, G. (2018). Overview on Microfluidic Reactors in Photocatalysis: Applications of Graphene Derivatives. Catal. Today 315, 79-92. doi:10.1016/j.cattod.2018.05.041

Zazueta, A. L. L., Destaillats, H., and Li Puma, G. (2013). Radiation Field Modeling and Optimization of a Compact and Modular Multi-Plate Photocatalytic Reactor (MPPR) for Air/water Purification by Monte Carlo Method. Chem. Eng. J. 217, 475-485. doi:10.1016/j.cej.2012.11.085
Zhang, W., Xiang, X.-X., Chen, J., Yang, C., Pan, Y.-L., Cheng, J.-P., et al. (2020). Direct C-H Difluoromethylation of Heterocycles via Organic Photoredox Catalysis. Nat. Commun. 11, 638-648. doi:10.1038/s41467020-14494-8

Zheng, L., Xue, H., Wong, W. K., Cao, H., Wu, J., and Khan, S. A. (2020). Cloudinspired Multiple Scattering for Light Intensified Photochemical Flow Reactors. React. Chem. Eng. 5, 1058-1063. doi:10.1039/d0re00080a

Zhou, M.-D., Peng, Z., Li, L., and Wang, H. (2019). Visible-light-promoted Organic Dye Catalyzed Perfluoroalkylation of Hydrazones under Mild Conditions. Tetrahedron Lett. 60, 151124. doi:10.1016/J.TETLET.2019.151124

Zhu, Z., Yang, L., Yu, Y., Zhang, L., and Tao, S. (2020). Scale-up Design of a Fluorescent Fluid Photochemical Microreactor by 3D Printing. ACS Omega 5, 7666-7674. doi:10.1021/acsomega.0c00511

Conflict of Interest: The authors declare that the research was conducted in the absence of any commercial or financial relationships that could be construed as a potential conflict of interest.

Publisher's Note: All claims expressed in this article are solely those of the authors and do not necessarily represent those of their affiliated organizations or those of the publisher, the editors, and the reviewers. Any product that may be evaluated in this article, or claim that may be made by its manufacturer, is not guaranteed or endorsed by the publisher.

Copyright $\odot 2022$ de Oliveira, Lira, Riella, Soares and Padoin. This is an open-access article distributed under the terms of the Creative Commons Attribution License (CC BY). The use, distribution or reproduction in other forums is permitted, provided the original author(s) and the copyright owner(s) are credited and that the original publication in this journal is cited, in accordance with accepted academic practice. No use, distribution or reproduction is permitted which does not comply with these terms. 